\title{
Transmitted reference UWB wireless receiver based on FFT technique
}

\author{
Rashid A. Fayadh ${ }^{1}$, Mousa K. Wali ${ }^{2}$ and Doaa Yousif Al_taee ${ }^{3^{*}}$ \\ Department of Electrical Engineering Technical College, Middle Technical University, Baghdad, Iraq ${ }^{1}$ \\ Dean of Electrical Engineering Technical College, Middle Technical University, Baghdad, Iraq ${ }^{2}$ \\ MSC Student, Department of Computer Engineering, Electrical Engineering Technical College, Middle Technical \\ University, Baghdad, Iraq ${ }^{3}$
}

\section{(C)2017 ACCENTS}

\begin{abstract}
The UWB system is very widely used in wireless communication, but it's had some challenges such as estimation channel, low power and low complexity. Use the UWB system in wireless communication can be high performance, better BER performance and very low complexity. In this paper, proposed a new system known low complexity transmitted reference ultra-wide band (LC-TR-UWB) based on fast Fourier transform (FFT). This system has advantages such as no need channel estimation and the reference pulse transmitter with data pulse and generate reference locally. This system may be used FFT to convert signals from time domain to frequency domain. Then, cut-off high frequencies that caused confusion the signal and finally use inverse fast Fourier transform (IFFT) to translate from the frequency domain to the time domain. The simulation results show the performance of this system has efficiency, more noise-resistant and BER better performance than previous systems.
\end{abstract}

\section{Keywords}

TR-UWB, FFT, IFFT, LC-TR-UWB.

\section{Introduction}

The UWB can spread to 20 meters (60 feet) with except able power and frequency range of it from 3.1 to $10.6 \mathrm{GHz}$. In recent years, the UWB system is continuously growing. In a wireless communication system, channels used in UWB system face problem $[1,2]$. This problem is channel estimation. There are some challenges for designing a receiver such as capture energy for data, channel estimation and generate a reference locally. So that, designing receiver is an expensive process and complicated design [3]. So that, propose a new system known TRUWB system. In this system, the reference pulse is transmitted with data pulse instead of generating this signal in the receiver [4]. TR-UWB no needs to estimate the wireless channel. In this work, propose a new system known LC-TR-UWB based on FFT for designing receiver will be low complexity from other systems and more noise resistance than TR-UWB and high efficiency and no need to channel estimation. Use FFT to convert signals from time domain to frequency domain then, remove high frequencies that caused noise and confuse for receiver processes that mixing many signals and then use IFFT to inverse converting this signal from frequency to time domain.

*Author for correspondence

136
Show in section two, study TR-UWB system while in section three, study FFT algorithm. In section four, propose the new system known LC-TR -UWB. In section five, show results and discussion. Finally, in section six, conclusions were qualified.

\section{TR-UWB system}

This system known as TR-UWB has some benefits such as low complexity compared with a rake receiver so it is called low complexity alternative of rake receiver [5]. In a transmitter, each frame of TRUWB consists of two types of pulses called reference pulse and data pulse. In reference pulse, no need to delay apparatus while data pulse need to delay apparatus. In this system, obtain on the data pulse after modulation (BPSK modulator) and the reference pulse without modulation. After that, multiplier both pulses to give the transmitter signal [6]. In channel model, the transmitted signal of this system enters to wireless channel known as AWGN channel which is added to channel model some noise signals to required transmitter signal [7]. In receiver, first either Amplifier or LPF is used to amplify this signal and LPF to reduce noise by cut-off high frequencies that caused noise and confusion. The reference pulse does not need to delay apparatus while data pulse needs to delay apparatus. Then, make correlator for both data 
pulse and reference pulse. The correlator signal goes to integrate and decision output circuit [8]. The block diagram of the TR-UWB transceiver is shown in Figure 1. The transmitted and received signals are expressed bellow:

$S(t)=\sum_{i=0}^{\infty} \sum_{j=0}^{N s-1}\left[u\left(t-N_{s} T_{f}-j T_{f}\right)+\right.$

$\left.\operatorname{diu}\left(t-i N_{s} T_{f}-j T_{f}-T_{d}\right)\right]$

Where,

$S(t)$ is transmitted signal, $T_{f}$ is frame duration, $d_{i}$ is tranamitted data

$N_{s}$ is number of samples, $u(t)$ is transmitted data $p u$

lse, Ep is energy per pulse

$T_{d}=T_{s} N_{f}$ is length of each bit data.

While equation of correlation output can be expressed as:

$Z(t)=\sum_{j=0}^{N s-1} \int_{i N s T f+j T f+T d}^{i N s T f+j T f+T d+\tau} \operatorname{ri}(t) r i\left(t-T_{d}\right) d t$

$=y(t)+n 1(t)+n 2(t)+n 3(t)$

Where

$Z(i)$ is correlation output, $\tau=$

is integration time, $y(t)$ is the use ful signal

$n_{1}(t), n_{2}(t), n_{3}(t)=$ noise .

And the equation at receiver output can be expressed as:

$r i(t)=\sum_{j=0}^{N s-1}[u(t-i N s T f-j T f)+\operatorname{diu}(t-$

$i N s T f-j T f-T d)]+n(t)$

Where

$\mathrm{ri}(\mathrm{t})$ is received signal, $\mathrm{n}(\mathrm{t})$ is zero mean gaussian $\mathrm{n}$.

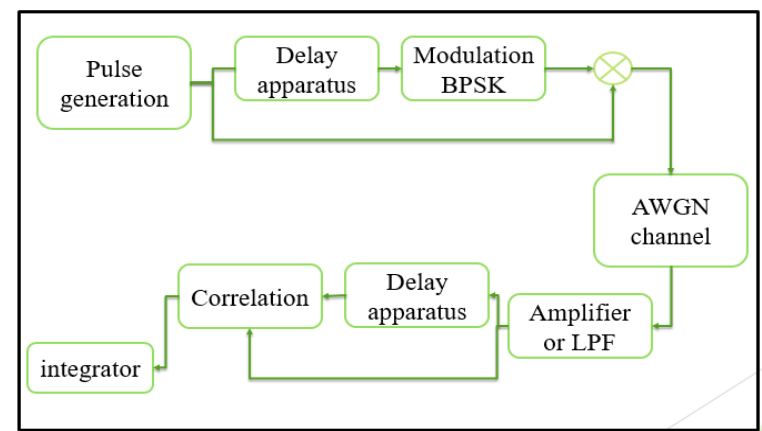

Figure 1 TR-UWB transceiver block diagram

\section{Fast Fourier transform (FFT)}

FFT plays a significant role in signal processing applications to transform the time domain signal to the frequency domain. FFT has several limitations, such as losing time domain information during the transformation, it should be applied to the entire length of the signal, and it does not allow the user to analyze the frequency changes of the input signal during any given time duration. The frequency spectrum does not show the time localization of frequency components in FFT [9]. Therefore, this transform is only useful for analyzing the stationary signal and non-stationary signals with some conditions. However, pre-windowing the signal provides time localization as a short time Fourier transform (STFT) method which is adopted by assuming that the signal is stationary within this short period of the window. The Fourier Transform is defined bellow [10].

$X(w)=\int_{-\infty}^{+\infty} x(t) e^{-j w t} d t$

The inverse Fourier Transform can express as.

$x(t)=\frac{1}{2 \pi} \int_{-\infty}^{+\infty} X(w) e^{j w} d w$

Where, $\mathrm{w}$ is the angular frequency, $\mathrm{x}(\mathrm{t})$ is the input signal and $\mathrm{X}(\mathrm{w})$ is the corresponding Fourier transformed signal.

\section{Low complexity TR-UWB receiver}

To make low complexity system, the following steps should be followed.

Step one, each frame of TR-UWB consists of two pulses called reference pulse and data pulse. Step two, in transmitter, the transmitted reference pulse passes directly while data pulse passes to delay apparatus and modulation. Get on data pulse after modulation while to get on TR-UWB transmitter signal after multiplying both of these pulses (reference and data pulses). Step three, in channel model, use an AWGN channel in this system because in all type modulator shows the BER performance of AWGN channel better than a Rayleigh fading channel. Step four, the output signal of channel model will be received by proposing receiver. In TRUWB receiver, the reference pulse passes directory without demodulation, while data pulse passes to demodulation circuit. Correlator both of these pulses (data and reference pulses) and then, the correlator output signal will be transform by FFT that convert signal from time domain to frequency domain to reduce noise must by cutoff frequencies that caused noise. FFT is used to remove high frequencies from a frequency domain signal to get rid of noise signal to make low complexity of the TR-UWB receiver [10]. Then supply this signal to IFFT to convert it from frequency domain to time domain. Therefore, this system is proposed to make system known LC-TRUWB based on FFT and get to receiver signal with very less noise and without confusing as show in Figure 2 and mathematical simulation can be expressed bellow [5]: Make FFT for received signal express bellow.

$X(w)=\int_{-\infty}^{+\infty} r i(t) e^{-j w} d t$ 
Rashid A. Fayadh et al.

Remove high frequency can be defining as.

$Y(w)=X(w)-n 1(t)$

(7)

Where, $\mathrm{n}_{1}$ is high frequencies. Make IFFT can express as.

$r i(t)=\frac{1}{2 \pi} \int_{-\infty}^{+\infty} Y(w) d w$

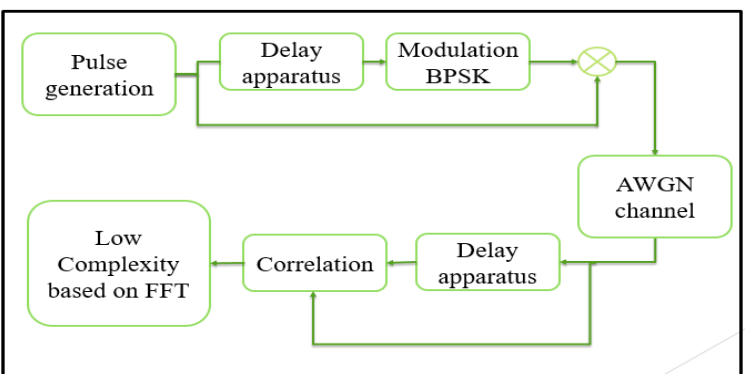

Figure 2 TR-UWB Based on FFT

\section{Results and discussions}

In this system, generate two types of pulse data and reference pulses and make modulation only for reference pulse. The output transmitter signal can show in Figure 3.

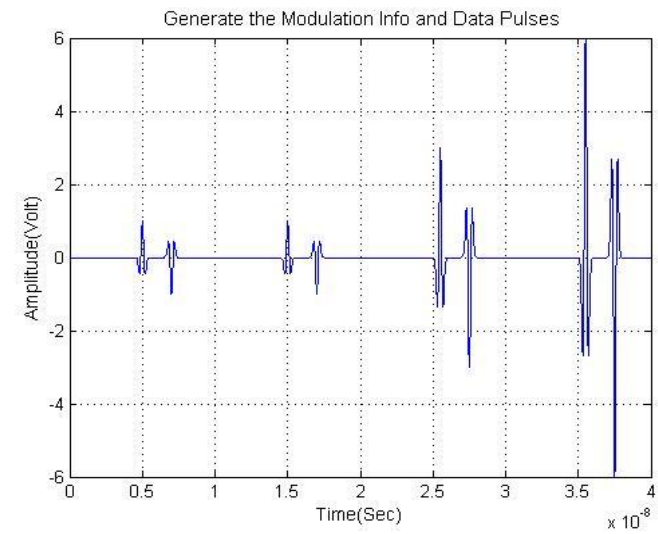

(a)

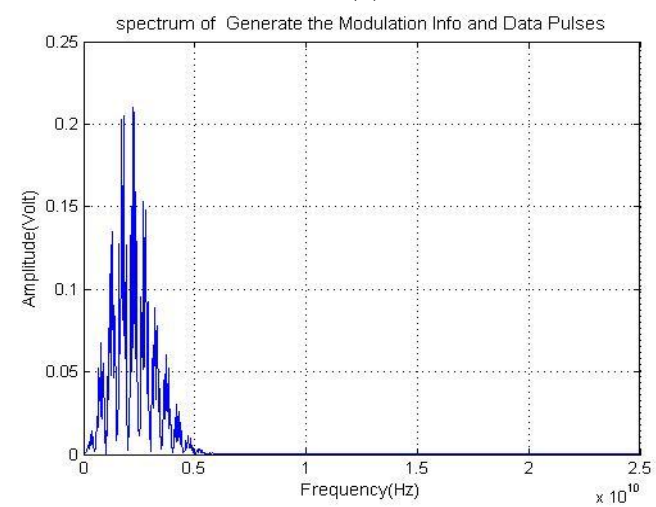

(b)

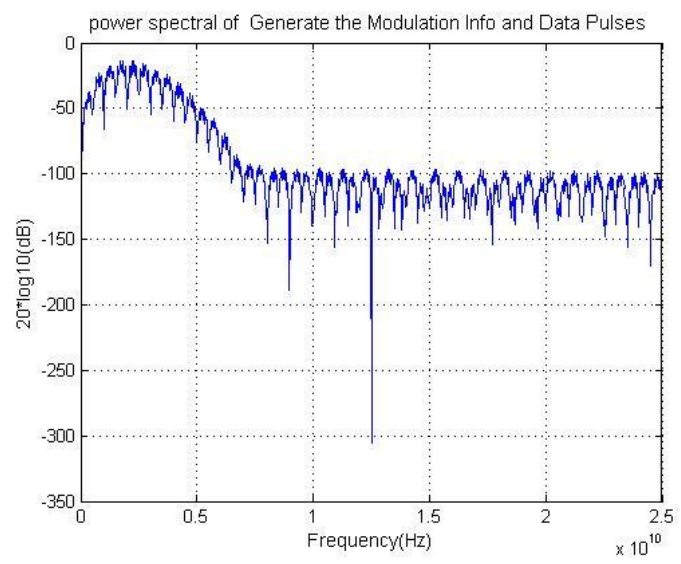

(c)

Figure 3 TR-UWB transmitter (a) The output signal, (b) Spectrum and (c) Power spectral

In addition, the noise signal of channel and the output signal from channel can show express in Figure 4.

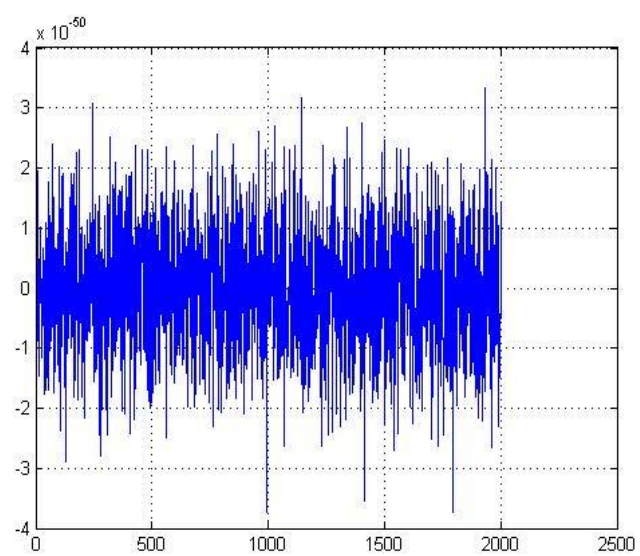

(a)

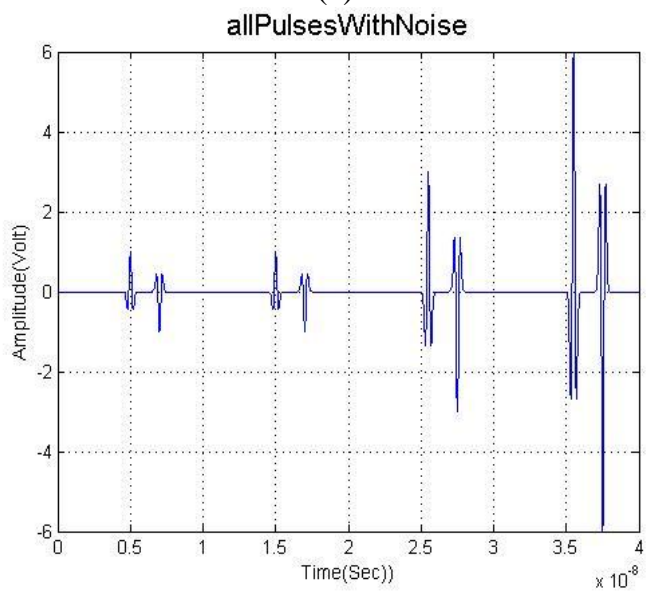

(b) 


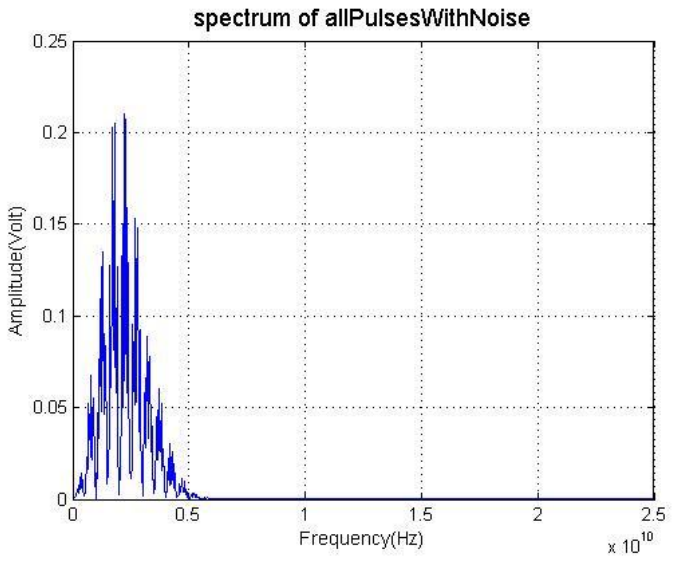

(c)

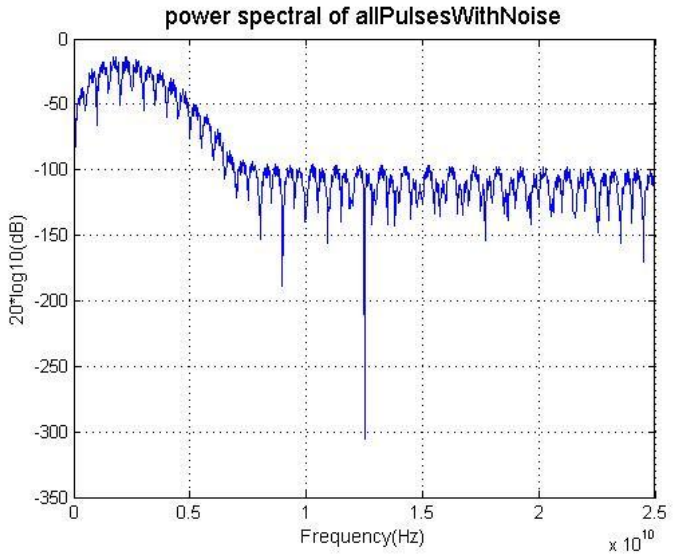

(d)

Figure 4 AWGN channel (a) The noise signal, (b) The output signal, (c) Spectrum and (d) Power spectral

The received signal with delay and correlator both reference and data pulses are shown in Figure 5.

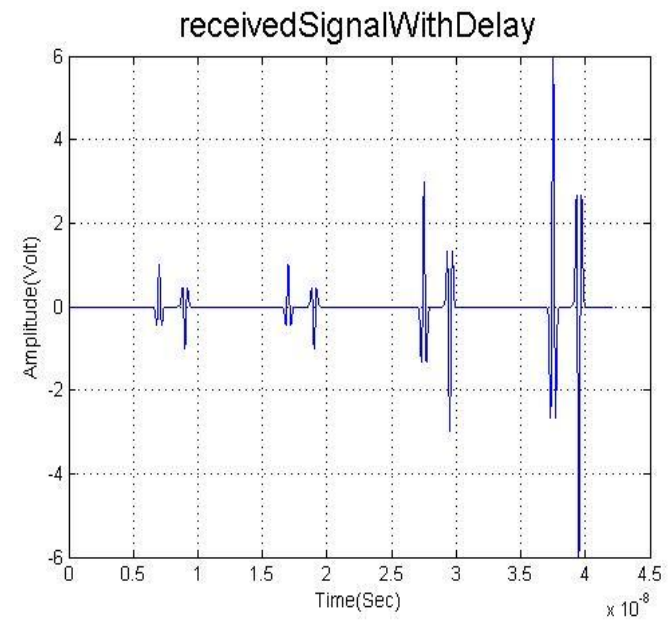

(a)

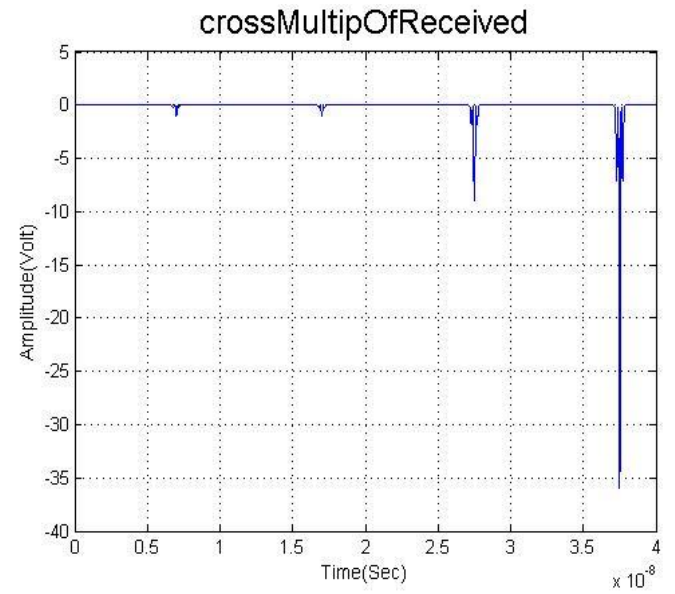

(b)

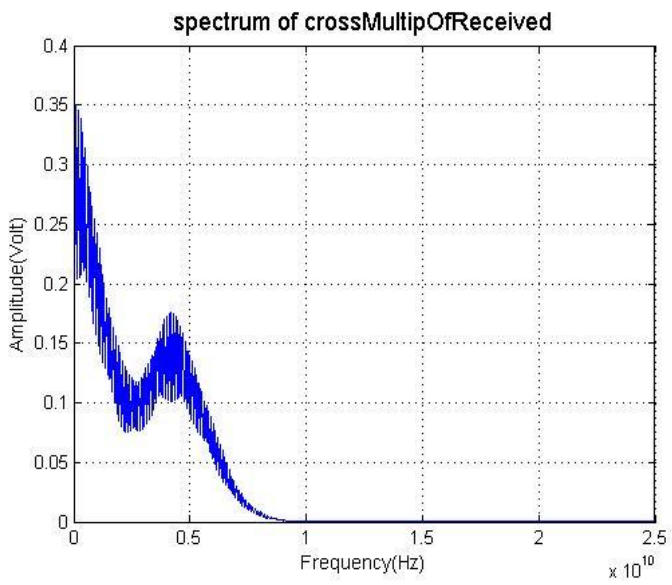

(c)

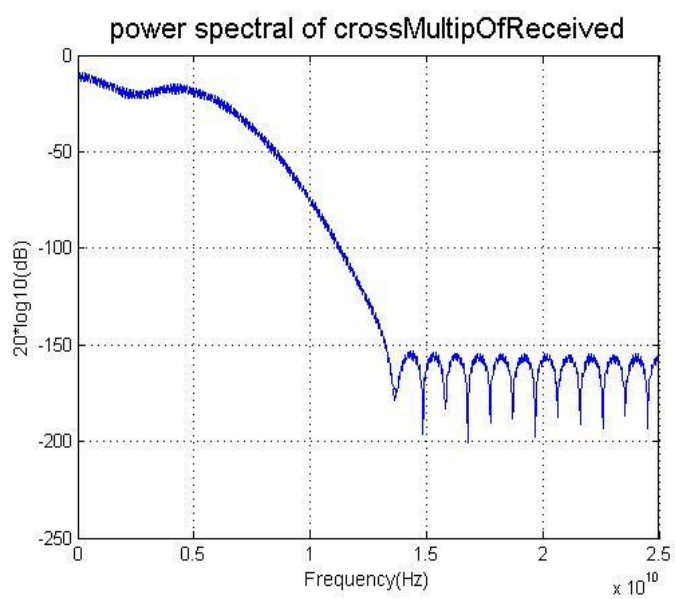

(d)

Figure 5 (a) The output received signal with delay, (b) The output correlator signal, (c) Spectrum of the output correlator signal and (d) Power spectral of the output correlator signal 
To reduce the noise of output received signal used FFT, remove high frequencies and IFFT. The output signal of LC-TR-UWB receiver is shown in Figure 6.

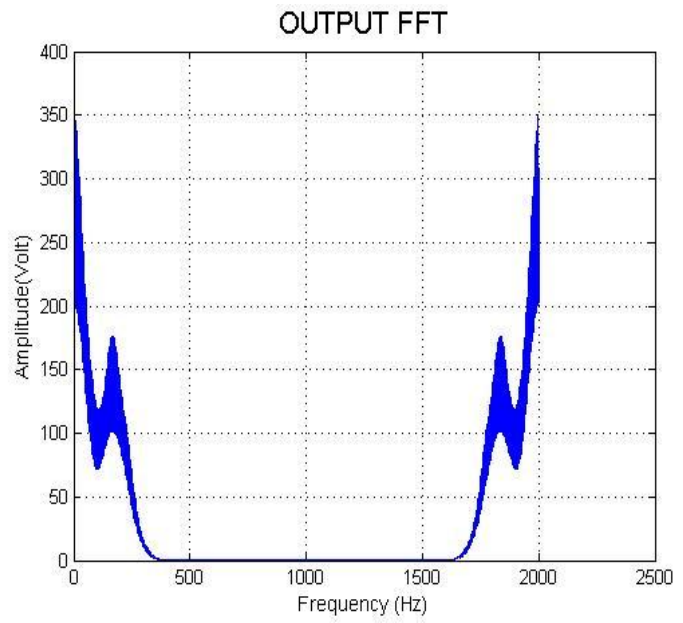

(a)

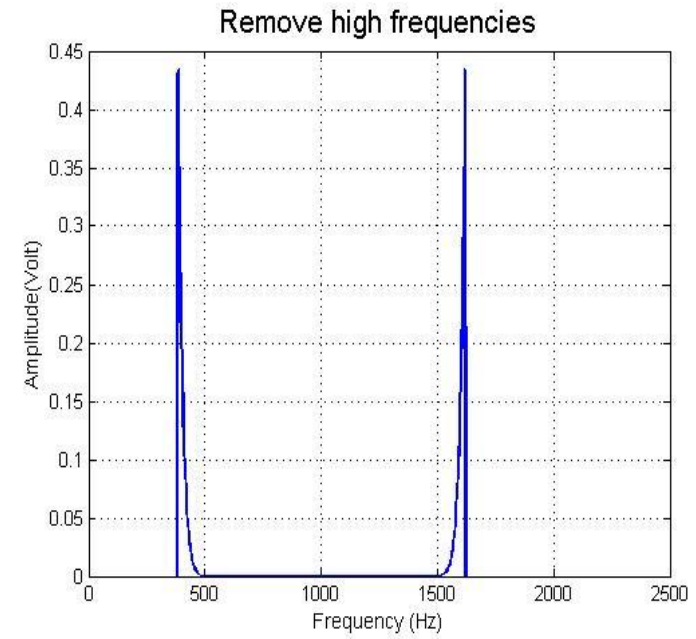

(b)

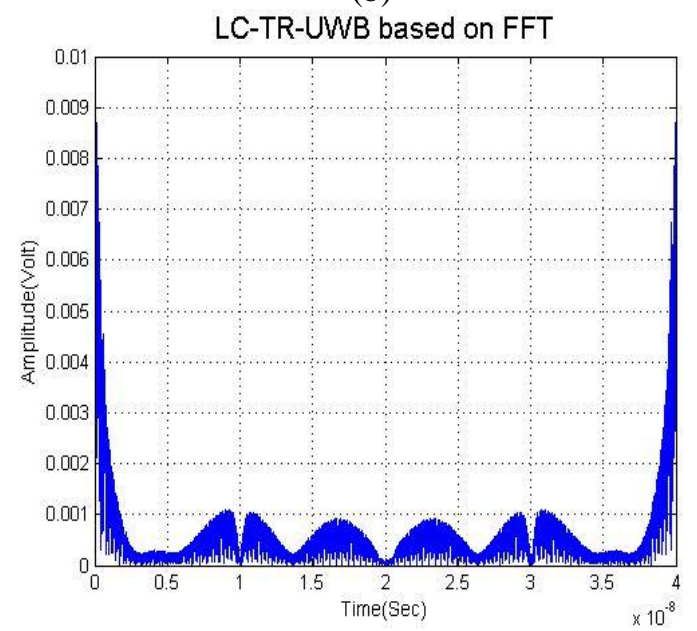

(c)

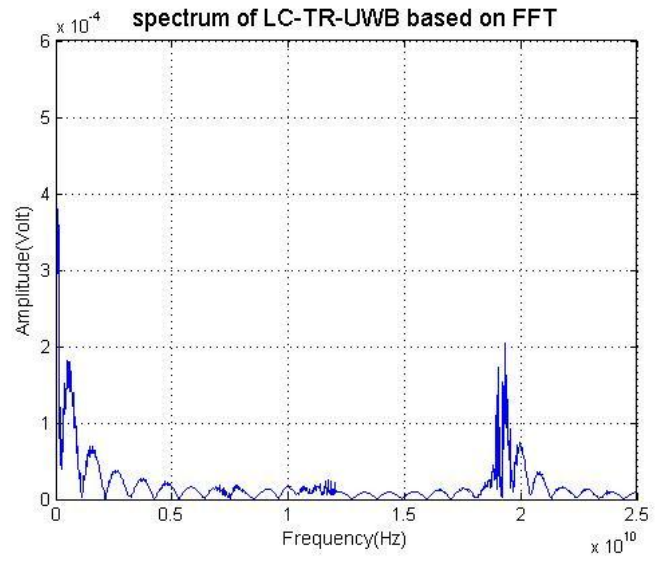

(d)

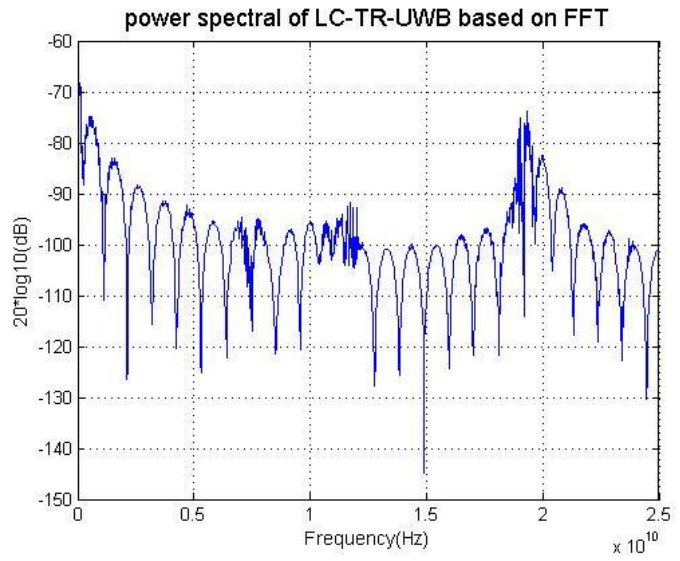

(e)

Figure 6 LC-TR-UWB receiver based on FFT, (a) the output of FFT, (b) High frequencies removal, (c) The output of IFFT, (d) Spectrum and (e) Power spectrum

Finally, The TR system has been analyzed numerically and by simulation. The AWGN standard channel models have been described and used to simulate real word system performance of the TR systems. It is paper that the TR system without channel estimation because of AWGN channel added the same noise for both pulses. In the receiver, The reference pulse has only noise signal while the data pulse has noise and information signal so that when subtracting the reference pulse from the data pulse obtaining on the transmitter signal. Also that, the LCTR system based on FFT is very useful for UWB application because all application needs to system with low cost, low weight, low complexity and addition to this system high performance and BER performance efficiency. It is shown in Figures and Table 1. This system is low power, low complexity, BER performance efficiency, high performance of this system and without channel estimation. Can 
show the value of BER will be minimize and reduce the wrong bits. In addition to, used FFT to translate the signal from time domain to frequency domain. Then, remove high frequencies that caused noise. After that, translate the signal from frequency domain to time domain.

\begin{tabular}{lll}
\multicolumn{3}{l}{ Table 1 LC-TR-UWB receiver based on FFT } \\
\hline $\begin{array}{l}\text { LC-TR-UWB } \\
\text { Receiver based } \\
\text { on FFT }\end{array}$ & Wrong BITS & BER Value \\
\hline TR-UWB receiver & 511.0712 bits & 0.2554 \\
LC-TR-UWB & 302.6867 bits & 0.1513 \\
receiver based on & & \\
FFT & & \\
\hline
\end{tabular}

\section{Conclusion}

From the simulation results in time and frequency domains of the traditional TR-UWB system, the received signal contains much noise. This noise caused by any error bits that leads to reduce transmission efficiency. In addition, the system is complicated in design because of amplification and noise reducing are taking place in this design the proposed LC-TR-UWB system that's based on FFT technique was simulated to show the modified results in terms of reducing noise, improved efficiency and low complexity these results were coming from very low error bits and less confuse.

\section{Acknowledgment}

None.

\section{Conflicts of interest}

The authors have no conflicts of interest to declare.

\section{References}

[1] Kim SD, Lee JH. A new transmitted-reference automotive UWB radar using unequaled amplitude. International Journal of Signal Processing, Image Processing and Pattern Recognition. 2009; 2(2):43-50.

[2] Farhang M, Salehi JA. Optimum receiver design for transmitted-reference signaling. IEEE Transactions on Communications. 2010; 58(5).

[3] Jin Y, Liu H, Kim KJ, Kwak KS. A reconfigurable digital receiver for transmitted reference pulse cluster UWB communications. IEEE Transactions on Vehicular Technology. 2014; 63(9):4734-40.

[4] Rissafi Y, Talbi L, Ghaddar M. Experimental characterization of an UWB propagation channel in underground mines. IEEE Transactions on Antennas and Propagation. 2012; 60(1):240-6.

[5] Tutay ME, Gezici S. Optimal and suboptimal receivers for code-multiplexed transmitted-reference ultra-wideband systems. Wireless Communications and Mobile Computing. 2013; 13(16):1435-49.

[6] Jin Y, Kwak KS. A transmitted reference pulse cluster averaging UWB receiver. IEEE Systems Journal. 2017; 11(2):1107-15.

[7] Wali M, Fayadh, Al_taee DY. Performance of AWGN and fading channels on wireless communication systems using several techniques. International Journal of Wireless Communications and Networking Technologies.2017; 6 (3):19-23.

[8] Choi JD, Stark WE. Performance of ultra-wideband communications with suboptimal receivers in multipath channels. IEEE Journal on Selected Areas in Communications. 2002; 20(9):1754-66.

[9] Rao KR, Kim DN, Hwang JJ. Fast Fourier transformalgorithms and applications. Springer Science \& Business Media; 2011.

[10] Kehtarnavaz N, Saki F. Anywhere-anytime signals and systems laboratory: from MATLAB to smartphones. Synthesis Lectures on Signal Processing. 2016; 8(1):1-210. 